\title{
ATLAS End Cap Toroid Cold Mass and Cryostat Integration
}

\author{
D. E. Baynham, F. S. Carr, E. Holtom, E. F. Towndrow, H. H. J. ten Kate, A. Dudarev, and J. Buskop
}

\begin{abstract}
The ATLAS experiment at LHC (CERN) will utilize a large, superconducting, air-cored toroid magnet system with a long Barrel Toroid and two End Cap Toroids. Each End Cap Toroid will contain eight racetrack coils mounted as a single cold mass in a cryostat vessel of approximately $10 \mathrm{~m}$ diameter.

This paper presents the design principles and realization of the cold mass assembly. The procedures for integration of the full cold mass, 120 tonnes, into the vacuum cryostat are described. The overall status of toroid magnet integration and planning for test and installation is reviewed.
\end{abstract}

Index Terms-Assembly, magnet, superconducting, toroid.

\section{INTRODUCTION}

$\mathbf{T}$ HE ATLAS muon spectrometer is based on a superconducting toroid magnet system [1] consisting of a long Barrel Toroid (BT) and two End Cap Toroids (ECT's). The major features of the End Cap Toroid Magnets have been described elsewhere [2], [3]. Each ECT consists of a cold mass, eight superconducting coil modules and eight keystone boxes, mounted in a large castellated cryostat [3]. The cold mass will be directly cooled by pumped circulation of two phase helium. All services will be carried through a single turret.

In this paper we describe the final industrial fabrication of major components, their delivery to CERN and the integration to form the final Cold Mass and Toroid assembly.

\section{COLD MASS FABRICATION IN INDUSTRY}

The Cold Mass consists of eight superconducting coils and eight keystone box modules. The keystone box modules are the structural elements that maintain the toroidal shape under gravitational and magnetic loads. The cold mass components were manufactured by BRUSH HMA BV in the Netherlands under a contract placed by NIKHEF, Amsterdam. The total fabrication required 16 coils and 16 keystone box (KSB) modules for the assembly of two complete toroids. Coil and keystone box fabrication has been described previously [2]. Industrial winding and impregnation of the coil modules was completed in April 2004. In parallel with the coil manufacture a test assembly of a full cold mass was made at the manufacturers works, see Fig. 1. The assembly was made with toroid axis vertical and confirmed the

Manuscript received September 20, 2005.

D. E. Baynham, F. S. Carr, E. Holtom, and E. F. Towndrow are with the CCLRC Rutherford Appleton Laboratory, Chilton, Didcot, Oxonshire OX11 0QX, U.K. (e-mail: d.e.baynham@ rl.ac.uk).

H. H. J. ten Kate, A. Dudarev, and J. Buskop are with CERN, CH-1211, Geneva 23, Switzerland.

Digital Object Identifier 10.1109/TASC.2005.864247

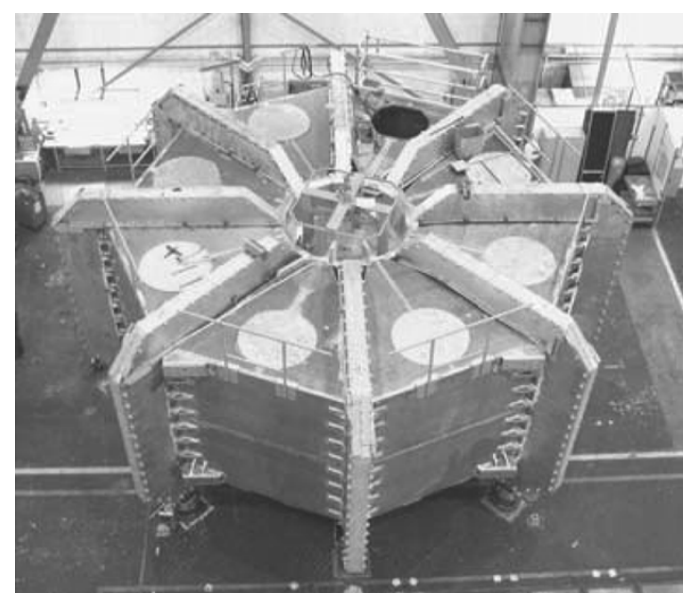

Fig. 1. Cold mass pre-assembly at manufacturers works.

feasibility of correct geometric assembly of coils and keystone box modules. Following this preassembly all coils and keystone boxes were transported to CERN.

\section{Cold Mass Assembly at CERN}

Since delivery of the cold mass components work has commenced at CERN to prepare modules and tooling for final cold mass assembly. Cold mass assembly at CERN is being made with the toroid axis horizontal see Fig. 2. Planning and tooling for cold mass assembly must be compatible with the final integration of the cold mass into the vacuum cryostat. For final integration a large tooling and infrastructure has been set up in building 191 at CERN. This will be described in Section IV. Cold mass assembly is being undertaken in four main phases:

\section{A. Preparatory Work on Coils and Keystone Boxes}

Extensive preparatory work has been carried out on both coils and keystone boxes. A key part of this work has been the preparation, installation and testing of cooling circuits on the coils and keystone boxes. Aluminum cooling circuits were installed in the coils at the fabrication stage. Since delivery, aluminum to stainless steel transitions have been welded on all coils in order to allow final manifold connections in stainless steel. Keystone box cooling circuits have been fabricated and installed, see Fig. 3. All circuits have undergone rigorous X-ray inspection, thermal cycling and leak testing.

Coil units have been prepared for installation of quench heaters thermal sensors, SQD's (Superconducting Quench Detectors) and voltage taps for quench detection and joint monitoring. 


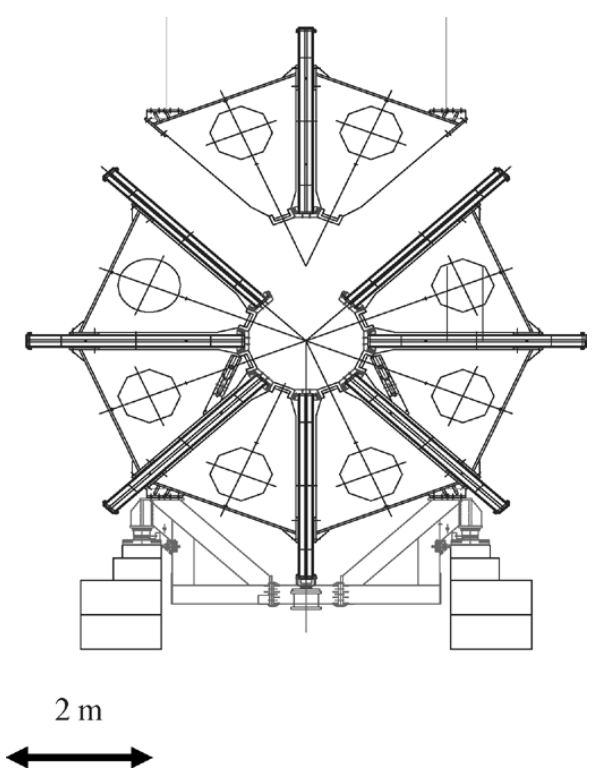

Fig. 2. Cold mass assembly at CERN.

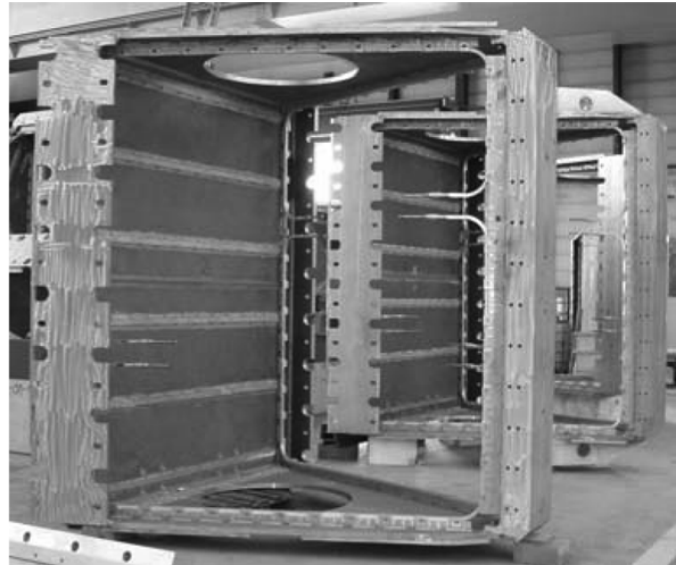

Fig. 3. Installation of keystone box cooling circuits.

\section{B. Coil/Keystone Box Alignment and Shimming}

When the coils and keystone boxes are assembled to form a complete cold mass it is important to locate the coils accurately at the $45^{\circ}$ azimuthal spacing and correct radial position in order to achieve accurate toroidal field profiles. It is also important to ensure correct mechanical connection interface between coils and keystone boxes, firstly, to transfer gravitational loads and, secondly, to transfer the magnetic loads. Fig. 4 shows the load transfer points at the inner circumference. At the design stage spaces were left, $5 \mathrm{~mm}$ at the inner radius and $10 \mathrm{~mm}$ at the outer radius. These spaces were designed to allow accurate geometric assembly taking account of the manufacturing tolerances. In order to complete the mechanical integrity of the assembly and allow uniform force transfer the spaces have been shimmed in preparation for the final assembly stage.

The shim process was carried out in two stages. The coils and keystone boxes were first aligned in a frame, see Fig. 5. In this process the coils were placed at accurate $45^{\circ}$ azimuthal and radial positions using pre-defined bushes.

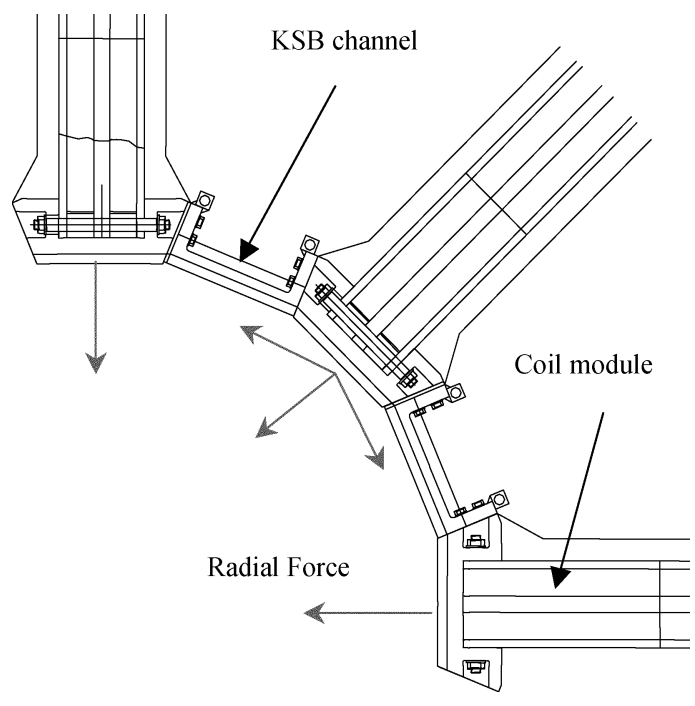

Fig. 4. Load transfer and shim positions at inner radius.

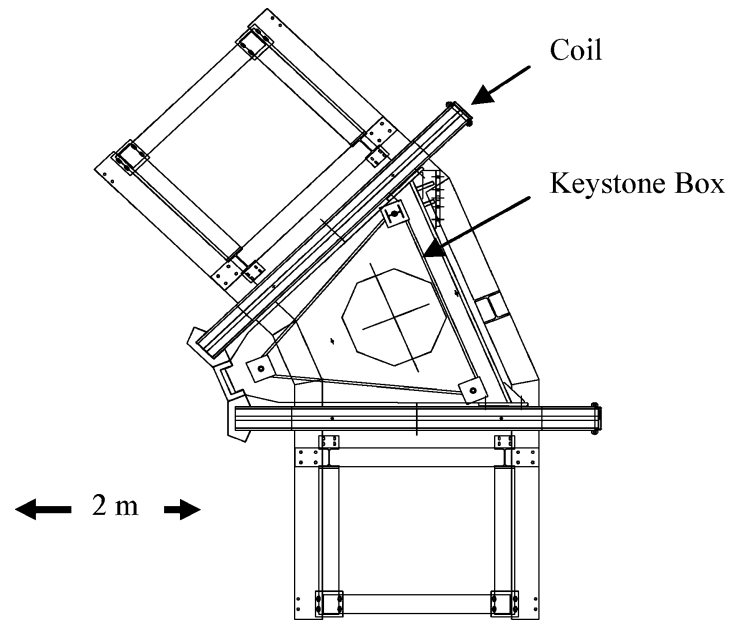

Fig. 5. Top view on coil and keystone box alignment frame.

With the coils set and the keystone box installed shim spaces were measured at localized positions on the main flange interfaces. This was repeated for all module positions.

The second stage was to prepare the shims over the extended flange faces. This was achieved by forming a sandwich of thixotropic epoxy and aluminum plates and deforming this to the correct interface thickness previously measured before curing the epoxy, see Fig. 6.

With this preparation procedure the coils and keystone boxes can be accurately re-assembled with axis horizontal to the defined geometry. Final bonding with a thin resin layer will ensure high quality force transfer surfaces.

All alignment and shim operations have been completed for the first toroid cold mass.

\section{Cold Mass Geometric Assembly}

A sequential build of the cold mass is now in progress. As an initial step the first coil (lower) was assembled with its two mating keystone boxes, see Fig. 7. Other sub-assemblies of coils and keystone boxes have been made in preparation for final assembly, see Fig. 8. 


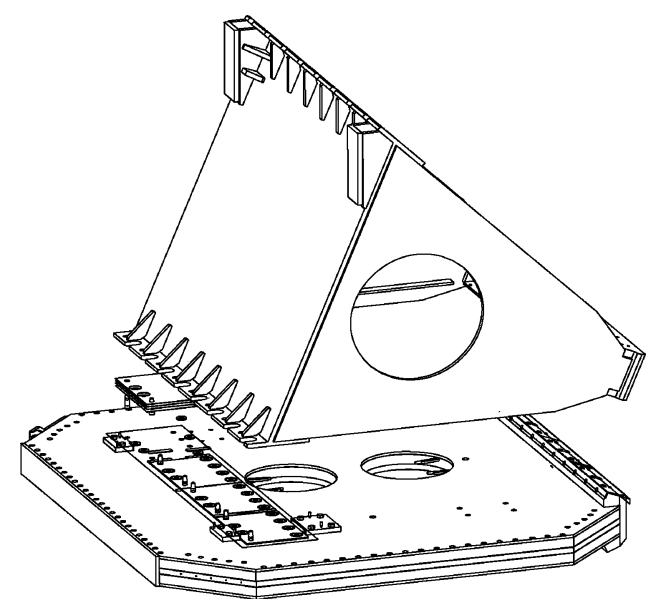

Fig. 6. Interface shim preparation procedure.

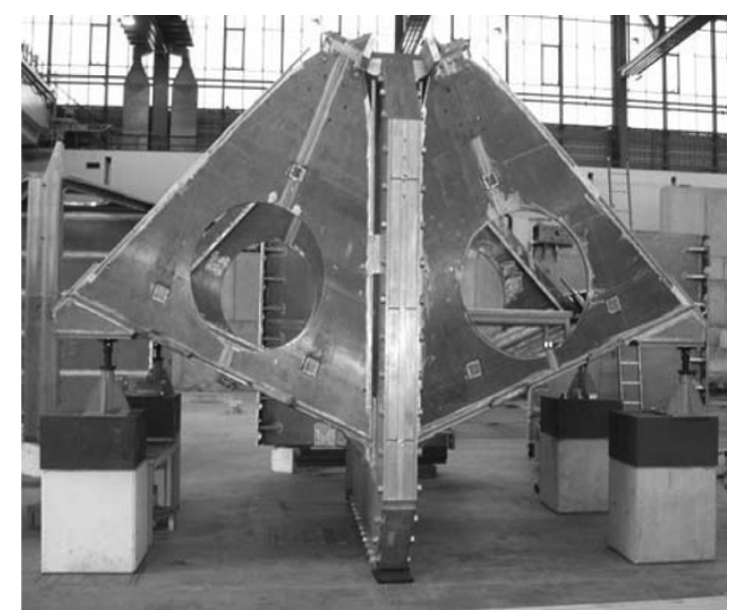

Fig. 7. Sub-assembly of lower coil with two keystone boxes.

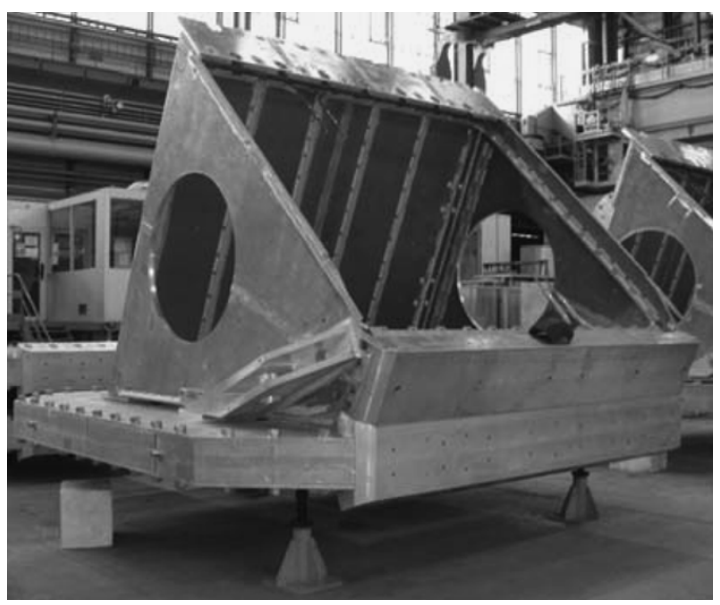

Fig. 8. Sub-assembly of coil with keystone box showing cold mass support.

For final assembly the first element will be transferred to the cold mass support frame on the toroid integration rails. Further sub-assemblies will be added, located by special blocks and finally glued and bolted at the flange interfaces. This sequence will continue until the mid plane coils (horizontal) are in place. A dry assembly of the top half of the cold mass will then be made. This dry assembly will allow in-situ confirmation of the

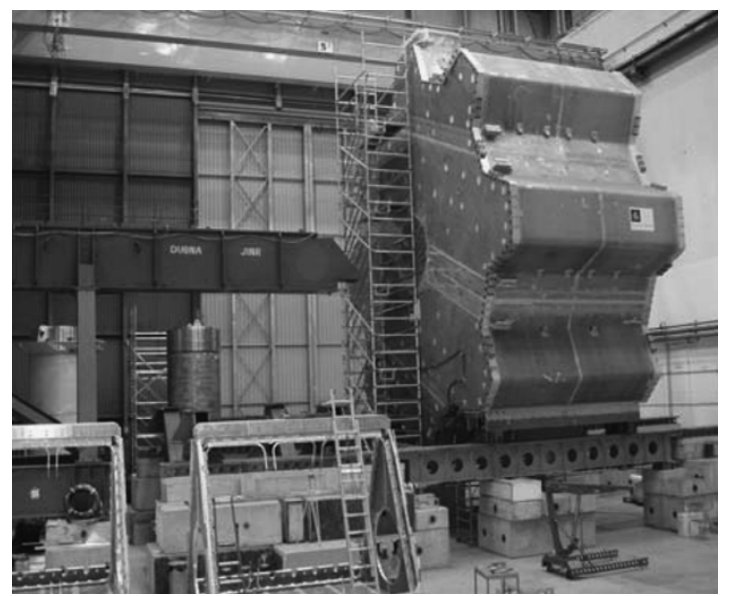

Fig. 9. End Cap Toroid integration infrastructure: vacuum vessel with air pads moving on rails in front of cantilever.

fit-up of the final parts in the keystone arch. Although careful alignment of the coils was made at the shimming stage some small geometrical errors will remain and could affect the positioning of the top coil in the arch. During this build up coil positions will be checked by survey.

When the position of the top coil is confirmed by this process final shim dimensions can be defined and implemented in a rebuild with final gluing and bolting of all elements.

When the cold mass is stripped back for rebuild of the top half the vacuum vessel bore tube and thermal shield will be installed in the cold mass bore.

\section{Instrumentation, Electrical Connection, Cooling Circuit Manifolding}

As the cold mass build progresses the electrical connections will be made between coils. A conductor tail from each coil will be formed across the end of the keystone box and the conductors welded to form the low resistance $\left(10^{-10} \mathrm{ohm}\right)$ connection. The joints will be clamped and bonded to the KSB structure to give mechanical and thermal stability.

The cooling circuits, 4 per coil, 4 per keystone box, will be connected through a stainless steel manifold system inside the keystone box structure. All manifolds have been preformed and assembled into sub elements for final installation, this will ensure a minimum number of in-situ welds in the keystone box structure. Manifold sub assemblies have been fully checked by $\mathrm{X}$-ray, thermal cycling and leak testing.

The cold mass will be fully equipped with instrumentation for temperature monitoring, quench detection, joint resistance monitoring and quench initiation. Quench initiation will be made by $2 \times 100 \mathrm{~W}$ heaters on each coil. These heaters will be bonded directly to the conductor feed and exit from the coil. All instrumentation is prepared and ready for installation.

\section{TOROID INTEGRATION}

When the cold mass assembly is complete the final stage will be the toroid integration i.e. installation of the cold mass in the cryostat. Transfer of the 130 tonne cold mass into the $10 \mathrm{~m}$ diameter vacuum vessel requires a set of heavy tooling which has been installed in B191 at CERN, see Fig. 9. The tooling comprises a large cantilever beam and a pair of assembly rails which allow the large components to be moved on air pads. 
The cold mass will be assembled on the mid point of the rails on a special support frame. When the cold mass is complete the frame with the cold mass will be moved over the large cantilever beam. At this stage the gravity support rods will be installed to connect the cold mass and centre bore tube. With the bore tube supported on the cantilever the full cold mass weight can be transferred to the cantilever and the support frame removed. With the end plates removed the vacuum vessel can be transferred over the cold mass using the rail and air pad system.

At this stage axial force transfer rods will be installed and load tested. The final thermal shield end plates will be fitted and electrical and pipe work connections made into the cryostat services turret. When this is complete turret services and vessel end plates will be fitted to complete toroid integration.

\section{SCHEDULE}

Assembly of the first cold mass for ECT A is scheduled for completion in November 2005. This will allow toroid integration to be completed early in 2006 . A pre-cooling test to $80 \mathrm{~K}$ is proposed before transfer to the ATLAS cavern in spring 2006.
The second toroid ECT C will follow with approximately 6 months interval.

\section{ACKNOWLEDGMENT}

We acknowledge the contribution of many work colleagues at Rutherford Appleton Laboratory and CERN in the realization and integration of the ATLAS End Cap Toroids.

\section{REFERENCES}

[1] H. H. J. ten Kate, "The superconducting magnet system for the ATLAS detector at CERN," IEEE Trans. Appl. Supercond., vol. 11, p. 347, 2001.

[2] D. E. Baynham et al., "ATLAS End Cap Toroid magnets cold mass design and manufacturing status," IEEE Trans. Appl. Supercond., vol. 14 , p. $485,2004$.

[3] — , "ATLAS End Cap Toroid magnets cryostat design, manufacture and integration at CERN," IEEE Trans. Appl. Supercond., vol. 14, p. 522,2004 